\title{
¿Antropología u ontología de la historia? La presencia de Carl Schmitt en la obra de Koselleck
}

\author{
Manuel Orozco Pérez \\ orozcoperezmanuel@gmail.com - https://orcid.org/0000-0002-6387-7825
}

\section{Fecha recepción: 29.05.2020 / Fecha aceptación: 25.09.2020}

\section{Resumen}

En este artículo se presentan los resultados de investigación de la estancia en el Nachlass Carl Schmitt que se encuentra en el Landesarchiv Nordrhein-Westfalen. El contenido de la correspondencia se ha organizado con vistas a ofrecer argumentos sólidos desde el punto de vista histórico a la tesis según la cual el proyecto de la historia conceptual

\section{Abstract}

This article presents the research results of the stay at the Carl Schmitt Nachlass located in the Landesarchiv Nordrhein-Westfalen. The content of the correspondence has been organized with a view to offering solid arguments from the historical point of view to the thesis that Reinhart Koselleck's project of conceptual history is not so much a method of his-

\footnotetext{
* Este artículo es un extracto revisado, corregido y ampliado de la tesis doctoral Temporalización y aceleración de la historia. La modernidad según Koselleck, que defendí en la Universidad Carlos III de Madrid en septiembre de 2017. Para su modificación he tenido en consideración la lectura de textos relevantes en el marco de la temática tratada, textos que por motivos de espacio y tiempo me fueron imposibles abordar en la tesis doctoral. Se da, asimismo, la azarosa circunstancia de que en la segunda quincena de diciembre de 2019, ya prácticamente finalizando el año, Jan Eike Dunkhase publicó para la editorial alemana Suhrkamp la correspondencia entre Carl Schmitt y Reinhart Koselleck, material éste que resultó fundamental para los resultados de mi investigación doctoral y que es indispensable para toda investigación orientada a la relación personal e intelectual entre Schmitt y Koselleck. La edición de Dunkhase incluye también varias cartas de Schmitt a Koselleck que tan sólo se encuentran en el Nachlass Reinhart Koselleck con sede en el Deutsche Literaturarchiv de la ciudad alemana de Marbach. R. Koselleck/C. Schmitt, Der Briefwechsel: 1953-1983, Berlín, 2019.
} 
Monográfico | ¿Antropología u ontología de la historia? La presencia de Carl Schmitt en la obra de Koselleck

de Reinhart Koselleck no es tanto un método de análisis histórico-filológico como una teoría de la modernidad. Asimismo, esta correspondencia sirve como punto de referencia para analizar la profunda relación personal e intelectual entre Koselleck y Schmitt.

\section{Palabras clave}

Reinahrt Koselleck, Carl Schmitt, Crítica y crisopibidis, dualismo político, historia conceptual torical-philological analysis as a theory of modernity. Furthermore, this correspondence serves as a point of reference to analyze the deep personal and intellectual relationship between Koselleck and Schmitt.

\section{Keywords}

Reinahrt Koselleck, Carl Schmitt, Critique and Crisis, political dualism, conceptual history 


\section{Crítica y crisis: una tesis doctoral, un libro}

Koselleck entra en el mundo académico con su tesis doctoral titulada Crítica y crisis. ${ }^{1}$ Considerada como la tesis en lengua alemana con mayor repercusión en el siglo XX dentro del ámbito de las ciencias humanas, ${ }^{2}$ las dudas de los dos examinadores ${ }^{3}$ sobre la calificación del trabajo -finalmente consiguió la segunda mejor calificación, magna cum laude- y el temor del propio Koselleck a que su trabajo no fuese aceptado por no cumplir con los cánones clásicos de la filosofía ni de la historia ni de la sociología, no hacían presagiar su posterior éxito. A lo largo de este ensayo se irán mostrando las semejanzas y diferencias entre la tesis y su posterior publicación en forma de libro. Este análisis se llevará a cabo a la luz de los planteamientos de Schmitt y las críticas de Johannes Kühn tal como aparecen reflejados en la correspondencia. La correspondencia entre Carl Schmitt y Reinhart Koselleck contenida en ese Nachlass está comprendida entre 1953 y 1983. Comienza con una carta de Koselleck datada el 21/01/53 y termina el 20/11/83, igualmente con una carta del historiador de Görlitz. La primera carta que encontramos de Schmitt data del 29/09/53 y la última del 04/03/79.

A finales de 1953 Koselleck tiene ante sí los exámenes orales final de carrera y la lectura de la tesis. En los exámenes orales Koselleck se examinó sobre cuestiones históricas de derecho constitucional (Staatsrecht) con Ernst Forsthoff -figura decisiva en Heidelberg para el

1. El título de la tesis iba acompañado de un subtítulo que sería modificado en su posterior publicación. Así, en la tesis puede leerse como subtítulo "Una investigación sobre la función política de la cosmovisión dualista en el siglo XVIII" (Eine Untersuchung der politischen Funktion des dualistischen Weltbildes im 18. Jahrhundert), mientras que en la publicación como libro reza "Un estudio sobre la patogénesis del mundo burgués” (Eine Studie zur Pathogenesen der bürgerlichen Welt).

2. Así lo recoge, sin citar la fuente, Christian Meier en su discurso pronunciado en un acto organizado en la Universidad de Bielefeld en conmemoración de la muerte de Koselleck: Ch. Meier, "Gedenkrede", en N. Bulst y W. Steinmetz (Dir.), Reinhart Koselleck 1923-2006. Reden zur Gedenkfeier am 24. Mai 2006, Bielefelder Universitätsgespräche und Vorträge 9, 2007, 7-34, aquí: 8; discurso recogido ahora también en H. Joas y P. Vogt (Dir.), Begriffene Geschichte, Berlín, 2011, 103-120, aquí: 103. Cf. J. W. Müller, A Dangerous Mind: Carl Schmitt in Post-war European Thought, New Haeven/London, 2003, 106.

3. La tesis fue defendida ante Karl Löwith y Johannes Kühn, quien, además de su tío materno, fue su director. 
restablecimiento académico de profesores cercanos tanto personal como ideológicamente a Carl Schmitt- ${ }^{4}$ con quien había hablado dos veces antes del examen; con Gadamer se examinó sobre Kant, estableciendo vínculos entre Descartes, Leibniz y Heidegger; y con su director de tesis, Johannes Kühn, sobre historia universal (Weltgeschichte), tomando a Alemania como ejemplo, y cuya divisa histórica rezaba «siempre demasiado tarde» (immer zu spät). Los exámenes orales, según le cuenta Koselleck a Schmitt, se desarrollaron en una atmósfera muy apacible. Las calificaciones finales fueron dos «magna» (con Forsthoff y Gadamer) y «summa» en historia (con Johannes Kühn). ${ }^{5}$

En cuanto a la tesis doctoral, ésta recibió finalmente, como se acaba de señalar, la calificación de magna cum laude.

La nota de mi tesis doctoral, dicho sea de paso, la bajó Löwith en su segundo informe en un momento en el que Kühn estaba dudando entre el summa y el magna cum laude, así que al final me otorgaron la segunda de estas calificaciones. Por aquel entonces yo personalmente estaba contento de que mi trabajo hubiese sido aceptado, ya que no podía caracterizarse claramente como un trabajo histórico ni sociológico ni tampoco filosófico. ${ }^{6}$

Crítica y crisis fue, en efecto, dirigida, al menos oficialmente, por el historiador Johannes Kühn, por quien Koselleck sentía un profundo respeto y admiración. En su discurso de agradecimiento (Dankrede) del 23 de noviembre del 2004 en conmemoración del quincuagésimo aniversario de su tesis doctoral, reconoce, quién sabe si quizá por deferencia hacia su maestro y, además, tío materno, que en pocas ocasiones había aprendido tanto en una hora como en la defensa de su tesis ante Kühn.

Sin haber acordado el tema, me preguntó en el debate dónde, cómo y por qué se había diferenciado la historia de Alemania de la de sus países vecinos. No se trataba de una inquisición moral para reformular ex post una nueva «vía especial» (Sonderweg), sino el intento sobrio de sondear las diferencias con ayuda de criterios comparativos comunes [...].?

En su tesis doctoral Koselleck se propuso analizar la función política de las tres Críticas kantianas. ${ }^{8}$ En un principio, su intención era titularla Dialéctica de la Ilustración. Tras

4. Cf. R. Mehring, Carl Schmitt. Aufstieg und Fall, Múnich, 2009, 464-468. Koselleck, junto a otras jóvenes promesas de la intelectualidad alemana, también visitaría los seminarios privados que Forsthoff organizaba en Ebrach, un pequeño pueblo de la Alta Franconia. Cf. D. van Laak, Gespräche in der Sicherheit des Schweigens, Berlín, 1993, 206; así como N. Sombart, Rendezvous mit dem Weltgeist, Fráncfort del Meno, $2000,205$.

5. R. Koselleck a C. Schmitt, 14/11/1954, en RW 265-8135. Ahora en R. Koselleck/C. Schmitt, Der Briefwechsel... op. cit., 66-73.

6. R. Koselleck, “Dankrede am 23. November 2004”, en S. Weinfuter (ed.), Reinhart Koselleck (1923-2006). Reden zum 50. Jahrestag seiner Promotion in Heidelberg, Heidelberg, 2006, 33-60, aquí: 50.

7. R. Koselleck, “Dankrede..., op. cit., 51.

8. R. Koselleck, “Dankrede..., op. cit., 34. 
hacerse eco de que en 1947 se habían publicado en Holanda unos fragmentos filosóficos con ese mismo título, ${ }^{9}$ se vio obligado a buscar uno nuevo. En efecto, Max Horkheimer y Theodor W. Adorno redactaron la Dialéctica de la Ilustración durante la II Guerra Mundial, entre 1939 y 1944. Se publicó «por primera vez en 1944 con ocasión del cincuenta cumpleaños de Friedrich Pollock en una pequeña edición [...] del Instituto de Investigación Social y, posteriormente, en Amsterdam, en 1947, en la imprenta de la editorial Querido, la más significativa del exilio alemán.» ${ }^{10}$ Volviendo a Crítica y crisis, su propio autor reconoce que se trata del «resultado de las sugerencias de Carl Schmitt comenzando por poner de relieve los análisis léxicos e investigando las transformaciones léxicas. Quizá se podría deducir una historia conceptual de la aplicación de los usos recíprocos de las palabras que hiciese referencia a la función política de la crítica y de la crisis.» ${ }^{11}$ En su epílogo a la reciente publicación de la correspondencia entre Koselleck y Schmitt, Jan Eike Dunkhase ha dejado poco margen para dudar que Koselleck encontrase el título de la tesis en un pasaje del texto de Schmitt Donoso Cortés in gesamteuropäischer Interpretation en el que habla de la «fatalidad que para la historia intelectual alemana de los dos últimos siglos vaya unida a los términos crítica y crisis.» ${ }^{12}$ Afirma Dunkhase que en la biblioteca de Koselleck se encuentra un ejemplar de esta obra adquirido en diciembre de 1950 -prácticamente en el momento de su publicación- en el que ambos conceptos aparecen doblemente subrayados. ${ }^{13}$ Por otro lado, se da la azarosa coincidencia de que allá por los años 30 del siglo pasado Bertolt Brecht y Walter Benjamin habrían planeado publicar una revista con el título Krise und Kritik, ${ }^{14}$ lo que parece indicar que el concepto de crisis lleva décadas siendo actual.

Existen, asimismo, otros motivos importantes a lo largo de la obra de Koselleck que van más allá de su tesis doctoral, que se forjaron en las clases magistrales a las que asistió en Heidelberg, y que serán decisivos en la formulación de sus fundamentos para una ontología de la historia orientada hacia la formación histórica de los conceptos. Entre ellos cabe destacar especialmente el estímulo de Ernst Wahle para concebir la historia más allá de las fuentes escritas. ${ }^{15}$ Tal premisa era, no obstante, un instrumento de análisis común en la investigación

9. Th. W. Adorno/Max Horkheimer, Dialéctica de la Ilustración. Fragmentos filosóficos, Madrid, 2009.

10. G. Schmid Noerr, "Nachwort des Herausgebers", en Max Horkheimer, Gesammelte Schriften, Band 5, Fráncfort del Meno, 2003, 424-457, aquí: 424.

11. R. Koselleck, “Dankrede..., op. cit., 64.

12. C. Schmitt, "Donoso Cortés in gesamteuropäischer Interpretation", en Donoso Cortés in gesamteuropäischer Interpretation. Vier Aufsätze, Colonia, 1950, 80-114, aquí: 100 (ejemplar anotado de la Bibliothek Reinhart Koselleck). Cita tomada de J. E. Dunkhase, "Nachwort. Asymmetrische Korrespondenz", en R. Koselleck/C. Schmitt, Der Briefwechsel... op. cit., 409-429, aquí: 417, nota 12.

13. J. E. Dunkhase, "Nachwort...”, op. cit., 417. En todo caso, esta intuición había sido puesta sobre la mesa hace ya más de tres lustros por J. W. Müller, A Dangerous Mind... op. cit., 266, nota 16.

14. E. Wizisla, “'Windschiefes', 'Grüppchenhaftes' und 'selbstverständliche Bedeutung. Das Zeitschriftenprojekt 'Krise und Kritik' (1930/31) aus der Sicht Ernst Blochs und die Edition der Dokumente”, en G. Klaus y L. Rehm, Global Benjamin. Vol. 2, Múnich, 1999, 801-809.

15. R. Koselleck, “Dankrede..., op. cit., 35. 
histórica que encontramos ya en otros autores que marcarían igualmente la línea de trabajo de Koselleck, entre los que destaca Otto Brunner.

El pensamiento teórico de Brunner se movía en círculos peculiares. Que los conceptos se pueden obtener únicamente de las fuentes es algo que él no creía. Para encontrar en las acciones lo que se anda buscando se necesitan primeramente categorías y conceptos sistemáticos del presente [...] Sin embargo, los conceptos modernos son sólo la condición, naturalmente la condición necesaria, sobre la que el historiador debe dar forma a conceptos individuales y a conceptos tipo que sean apropiados para el estudio del objeto histórico en cuestión. ${ }^{16}$

Décadas más tarde, Koselleck, por su parte, mantendrá una posición semejante, a saber: que la interpretación de la realidad histórica se decide no sólo sobre el terreno del control metódico de las fuentes, sino allí donde se procura articularlas lingüísticamente. ${ }^{17} \mathrm{Y}$ es que, en el fondo, una de las tareas esenciales de la historia de los conceptos en cuanto disciplina es hacer que el historiador sepa de su propio lenguaje, un lenguaje que no se encuentra necesariamente en las fuentes a partir de las cuales elabora su relato. Es necesario señalar, empero, una diferencia fundamental entre las posiciones de ambos historiadores respecto a la comprensión de la noción de Grundbegriff.

Lo que es un concepto histórico fundamental lo definió Koselleck en el primer volumen [de los Geschichtliche Grundbegriffe]. La distancia con el concepto histórico fundamental del Brunner de los años treinta como expresión de «orden concreto», que debe construir puentes entre el presente y la Edad Media, es evidente. Koselleck evita dar una definición donadora de sentido. Se trata de «conceptos rectores del movimiento histórico, un movimiento que en el transcurso del tiempo ha ido constituyendo el objeto de la investigación histórica.» ${ }^{18}$

Otro ejemplo de la influencia de los primeros años de estudio en Heidelberg es la noción de patogénesis que encontramos en el subtítulo de la obra publicada: Un estudio sobre la patogénesis del mundo burgués. La inspiración para este concepto la encontró en las clases magistrales del médico Viktor von Weizsäcker: «Cuyas espontáneas y, sin embargo, lógicamente planteadas clases magistrales sobre antropología médica, lo que para él significaba siempre antropología social, me indujeron a utilizar la metáfora de patogénesis del mundo burgués como subtítulo de la edición impresa.» ${ }^{19}$ Un estudio sobre la patogénesis del mundo

16. Th. Etzemüller, Sozialgeschichte als politische Geschichte, Múnich, 2001, 72.

17. R. Koselleck, "Fiktion und geschichtliche Wirklichkeit”, en Vom Sinn und Unsinn der Geschichte, Berlín, 2014, 80-95, aquí: 93.

18. Ch. Dipper, “Die 'Geschichtliche Grundbegriffe'. Von der Begriffsgeschichte zur Theorie der historischen Zeiten”, en H. Joas y P. Vogt (Dir.), Begriffene... op. cit., 288-316, aquí: 298. Cf. T. Pankakoski, “Conflict, Context, Concreteness. Koselleck and Schmitt on Concepts”, Political Theory, 6, 2010, 749-779, aquí: 754.

19. R. Koselleck, “Dankrede...”, op. cit., 35. 
burgués fue, en efecto, el subtítulo de la tesis publicada como libro. El subtítulo de la tesis doctoral propiamente dicha rezaba Una investigación sobre la función política de la cosmovisión dualista en el siglo XVIII (Eine Untersuchung der politischen Funktion des dualistischen Weltbildes im 18. Jahrhundert). ${ }^{20}$ El cambio en el subtítulo de la obra obedece a una serie objeciones de su director de tesis. Johannes Kühn propone a Koselleck que, además de deshacerse de la noción de burguesía (Bürgertum), evite en la medida de lo posible el término dualismo, ya que tal concepto era propio de la historia de las religiones y, por consiguiente, no debía aplicarse a las tendencias políticas del siglo XVIII.

En el curso de sus objeciones [Johannes Kühn] realizó dos importantes propuestas para las modificaciones. En primer lugar, tendría que precisar mis análisis en el sentido de que no debería hablar de burguesía, cuyo concepto estaría puesto en cuestión, sino del papel de la inteligencia y la espiritualidad burguesa. En segundo lugar, me ha planteado una objeción importante en cuanto al uso del concepto de dualismo. Este concepto pertenecería a la historia de las religiones y en vista de las tendencias totalitarias del siglo XVIII, que no son dualistas, sería inapropiado aplicarlo. ${ }^{21}$

Cómo poder evitar o substituir el concepto de dualismo era a finales de 1954 toda una incógnita. Es en la publicación de la tesis como libro donde se puede apreciar el trabajo de Koselleck para superar la noción de dualismo sin desestructurar las tesis fundamentales de su trabajo para obtener el título de doctor.

En la obra publicada (1959), Koselleck limitará el uso de la expresión «dualismo moral» -si bien no la abandona del todo- y hará más hincapié en el dualismo entre moral y política. De hecho, en ciertos pasajes de la tesis doctoral (1954) en los que utilizaba la expresión die moralischen Dualismen («los dualismos morales»), aparecen en la publicación reformulados como die Dialektik von Moral und Politik («la dialéctica entre moral y política»). Así, donde en la tesis doctoral se puede leer "Los dualismos morales" le confieren a la lucha una radicalidad que aún no correspondía en absoluto a la importancia de la burguesía alemana en su conjunto», 22 en la versión publicada encontramos «"La dialéctica entre moral y política" le confiere a la lucha una radicalidad que aún no correspondía en absoluto a la importancia de la burguesía alemana en su conjunto.» ${ }^{23}$ Tal cambio para la publicación se encuentra consecuentemente corregido en el mismo índice.

20. I. Nagel, “Der Kritiker der Krise. Zum 50. Jahrestag von Reinhart Kosellecks Promotion”, en H. Joas y P. Vogt, Begriffene... op. cit., 94-102, aquí: 99.

21. R. Koselleck a C. Schmitt, 05/11/1954, en RW 265-8138. Ahora en R. Koselleck/C. Schmitt, Der Briefwechsel... op. cit., 79.

22. R. Koselleck, Kritik und Krise. Eine Untersuchung der politischen Funktion des dualistischen Weltbildes im 18. Jahrhundert. Tesis doctoral, Heidelberg, 1954, 94. La cursiva en la cita es nuestra: «Die moralische Dualismen verleihen dem Kampf eine Radikalität, die dem sozialen Gewicht des deutschen Bürgertums insgesamt noch keineswegs entsprach.»

23. R. Koselleck, Kritik und Krise. Eine Studie zur Pathogenesen der bürgerlichen Welt, Fráncfort del Meno, 2013, 106. La cursiva en la cita es nuestra: «Die Dialektik von Moral und Politik verlieh dem Kampf eine Radikalität, die dem sozialen Gewicht des deutschen Bürgertums insgesamt noch keineswegs entsprach.» 
Mas ni siquiera es necesario llegar al primer capítulo para percibir las sutilezas del cambio. Éstas se muestran ya en las primeras líneas de la introducción. La tesis comienza así: «Desde un punto de vista histórico, la crisis actual de la historia mundial, políticamente determinada por la tensión existente entre los polos opuestos que representan Estados Unidos y Rusia como las dos potencias mundiales, es el resultado de la expansión europea sobre la faz de la tierra.» ${ }^{24} \mathrm{Y}$ estas son las primeras líneas del libro: «Desde un punto de vista histórico, la crisis mundial actual, determinada por la tensión existente entre los polos opuestos que representan Estados Unidos y Rusia, es el resultado de la historia europea. ${ }^{25}$ En ambos fragmentos el concepto de crisis juega ciertamente un papel fundamental; sin embargo, su genitivo es formulado de manera bien diferente: en el primero se trata de la crisis de la historia mundial; en el segundo, de la crisis global. No hay, empero, elementos suficientes para dudar de que, si bien formulados de un modo diferente, ambos genitivos hagan referencia a la situación global durante el primer y el segundo lustro posteriores la II Guerra Mundial. El patetismo de esas primeras líneas de la tesis contrasta con lo que hoy llamaríamos una cierta corrección política del libro, sin que por ello sus argumentos dejen de ser, a su manera, provocadores. Esta reformulación no es en modo alguno baladí. Koselleck está buscando un espacio que le permita abundar en la idea de división entre moral y política, un espacio que la estructura original de la tesis no le deja. Por eso se ve obligado a añadir páginas, siete en concreto, al inicio del primer capítulo, ${ }^{26}$ donde se pueden apreciar las sugerencias que le hizo Johannes Kühn el día de la defensa de la tesis.

Repárese ahora en las significativas modificaciones de los títulos que aparecen en ambos índices. El apartado IV del segundo capítulo se corresponde en la tesis doctoral con el apartado III del segundo capítulo. El título de éste último reza: «Die verborgene Wendung gegen den Staat: Die politische Funktion des Logenarcanus (Lessing)/Plannung heimlicher Gewaltsame (Illuminaten), aber Verdeckung ihrer poltischen Bedeutung "durch den moralischen Dualismus". ${ }^{27}$ En la publicación encontramos «Die verborgene Wendung gegen den Staat: Die politische Funktion des Logenarcanus (Lessing)/Plannung heimlicher Gewaltsame (Illuminaten), aber Verdeckung ihrer politischen Bedeutung "durch den Dualismus von Moral und Politik”. ${ }^{28}$ El cambio en el título del tercer capítulo es también muy significativo. En la tesis reza: «Die Dialektik des moralischen Dualismus und die politische Krise»; en el libro: "Krise und Geschichtsphilosophie».

Parece, por tanto, que el dualismo moral del que hace uso Koselleck en su tesis doctoral está más cerca de los planteamientos de Schmitt que de los de su director de tesis. Recordemos que, como ya se ha señalado un poco más arriba, uno de los aspectos que Johannes

24. R. Koselleck, Kritik und Krise. Eine Untersuchung... op. cit., 1.

25. Reinhart Koselleck, Kritik und Krise. Eine Studie... op. cit., 1.

26. Véase especialemente R. Koselleck, Kritik und Krise. Eine Studie... op. cit., 12.

27. R. Koselleck, "Inhaltsverzeichnis", en Kritik und Krise. Eine Untersuchung... op. cit. La cursiva en la cita es nuestra.

28. R. Koselleck, "Inhalt”, en Kritik und Krise. Eine Studie... op. cit. La cursiva en la cita es nuestra. 
Kühn consideró necesario de ser revisado fue precisamente el concepto dualismo moral por pertenecer a la historia de las religiones, no siendo así apropiado para explicar las tendencias totalitarias del siglo XVIII, que en sí mismas no eran dualistas. ${ }^{29}$ Pero también el concepto de «dualismo entre moral y política» que usa Koselleck para evitar el de «dualismo moral» sigue siendo un término puramente schmittiano. ${ }^{30}$ No obstante, habría espacio aquí para matizar este punto en concreto argumentando que tal dualismo podría remontarse hasta Maquiavelo y sus epígonos, con lo cual la crítica de Schmitt a la razón de Estado, en su tratamiento por Meinecke, podría no identificar inequívocamente la traslación directa del motivo de éste a Koselleck. En efecto, la contraposición entre política y moral irrumpe fuertemente en el Renacimiento como una contraposición entre «la moral cristiana y la praxis de aquellos que ejercen la acción política». ${ }^{31}$ Y Maquiavelo, o la lectura maquiavélica de Maquiavelo, puede ser ejemplo de ello. ${ }^{32}$ Llama la atención, empero, que Koselleck no se haya remitido a Maquiavelo a la hora de tratar de manera específica la problemática del dualismo entre la moral y la política. Esto no se debe en modo alguno al hecho de no haber tenido ocasión para hacerlo. Leyendo con detenimiento Crítica y crisis, se observa que su autor menciona a Maquiavelo -sin ni siquiera citar alguna de sus obras más conocidas- una única vez en relación al temor de los políticos ante el secreto de las logias masónicas. ${ }^{33}$ Oportunidad ésta que habría sido de lo más oportuna para remitirse a este supuesto origen del concepto «dualismo entre moral y política». Pero no lo hace. De ahí que, a nuestro entender, siga teniendo más peso el argumento de que el término procede de Schmitt, especialmente si se tiene en cuenta la lectura de la correspondencia entre ambos autores y las remisiones a los textos correspondientes.

Crítica y crisis muestra, por tanto, hasta qué extremo Koselleck conocía los postulados de Schmitt y su valoración de los trabajos de Meinecke. Es más que probable que leyese la recensión del jurista alemán sobre La idea de razón de Estado en la Edad Moderna, ${ }^{34}$ en la que, para refutar el planteamiento de esta obra, el jurista alemán intenta construir un argumento sobre la base de su teoría decisionista del estado de excepción que le permite dirigir una crítica feroz al planteamiento de Meinecke, tildándolo -iqué curioso!- de «dualismo moral». Meinecke excluye la idea de una línea continua de desarrollo, así como la de una acentuación de la dialéctica y contiene un dualismo moral que mantiene el equilibrio en elementos con-

29. R. Koselleck a C. Schmitt, 05/11/1954, en RW 265-8138. Ahora en R. Koselleck/C. Schmitt, Der Briefwechsel... op. cit., 69.

30. C. Schmitt a R. Koselleck, 11/11/1953, en RW 260-386. Ahora en R. Koselleck/C. Schmitt, Der Briefwechsel... op. cit., 31-33.

31. N. Bobbio, Teoría general de la política, Madrid, 2005, 200.

32. N. Bobbio, La teoría de las formas de gobierno en la historia del pensamiento político, México D.F., 2006, 70-71.

33. R. Koselleck, Kritik und Krise. Eine Studie... op. cit., 67.

34. C. Schmitt, "Zu Friedrich Meineckes 'Idee der Staatsräson"' Archiv für Sozialwissenschaft und Sozialpolitik, 56, 1926, 226-234. 
trapuestos. Este dualismo aparece ora como la contraposición entre ser y deber, ora como la contraposición entre poder y moralidad, ora en otras formas. ${ }^{35}$

El trasfondo de esta crítica es el marco liberal en el queda delimitado el análisis de Meinecke:

Su dualismo evita tanto la [...] parte lógico-metafísica como la parte jurídica del problema y se mantiene en lo moral, es decir, en la tradición liberal de los siglos XVIII y XIX. [...] Al Estado [...] le corresponde detentar el poder. Pero ese poder debe manifestarse en la esfera de lo ético y vincularse ahí con algo ajeno a su naturaleza, incluso contradictorio. ${ }^{36}$

También podría pensarse que, amén de este resquemor de Schmitt ante toda forma de liberalismo, el jurista alemán consigue vengarse aquí de un supuesto silencio de la MeineckeSchule sobre su Politische Romantik publicada en 1919, ${ }^{37}$ aunque las reticencias entre ambos autores no deben reducirse únicamente a este hecho. En una carta de Schmitt al historiador y politólogo alemán Waldemar Besson confiesa que su crítica a Meinecke

No llevaba mala intención. Yo se la envié por aquel entonces, en 1926, desde la más pura imparcialidad y con todo el respeto, para mí era lo más natural del mundo que así fuera; puesto que Meinecke era doctor honoris causa de la Facultad de Derecho de Bonn, a la que, a la sazón, yo pertenecía. Sin embargo, él me respondió con una breve carta en la que se mostraba ofendido y con la que rompía todo contacto posterior. ${ }^{38}$

Continuando con el trazo de la relación entre el Koselleck de Crítica y crisis y los planteamientos antiliberales de Schmitt, cabría señalar las siguientes líneas escritas por el este último en la recensión sobre el mencionado libro de Meinecke que hoy día podrían servir incluso de abstract de la tesis doctoral de Koselleck:

Desde el punto de vista histórico surge otra duda. ¿Es la realmente la idea de razón de Estado una idea fundamental apropiada para sustentar una interpretación comprehensiva del problema del Estado y del poder del siglo pasado? ¿Dado su sentido específico, no se encuentra anclada en una época determinada, en la del absolutismo de los siglos XVI y XVII, y es demasiado poco característica y central como para que una exposición histórica pudiese basarse en ese concepto? ${ }^{39}$

35. C. Schmitt, “Zu Friedrich Meineckes...”, op. cit., 227. La cursiva en la cita es nuestra.

36. C. Schmitt, “Zu Friedrich Meineckes...”, op. cit., 228.

37. R. Mehring, Carl Schmitt... op. cit. 197. No obstante, en una carta a Koselleck con fecha de 28/07/1966, Schmitt afirma haber conversado con Meinecke sobre su Politische Romantik, «lamentablemente de manera estéril», R. Koselleck/C. Schmitt, Der Briefwechsel... op. cit., 204.

38. C. Schmitt a W. Besson, 07/05/1959, en RWN 260-386, 16. Carta sin publicar.

39. C. Schmitt, “Zu Friedrich Meineckes...”, op. cit., 232. 
Otra sentencia relevante de la recensión que demuestra el interés de Schmitt por la historia de los conceptos es cuando afirma que «la historia de la palabra Estado no está escrita todavía» (die Wortgeschichte von Staat ist noch nicht geschrieben). Un concepto de Estado cuya especificidad, según Schmitt, ignora Meinecke, a saber: la esfera de la publicidad en contraposición a todo lo privado y a todo lo económico. ${ }^{40}$

\section{El argumento de Crítica y crisis}

En lo concerniente propiamente a la temática del libro, el hilo conductor que lo vertebra es el surgimiento del Estado absolutista moderno tras refrenar y superar las guerras de religión y su precipitada disolución con el estallido de la Revolución francesa. La Ilustración encontró su punto de partida en el Estado absolutista, del mismo modo que el Estado absolutista lo encontró en las guerras de religión, pero la Ilustración no interpretó el Estado absolutista como una respuesta a las guerras de religión. Una vez que estas finalizaron, fue visto como un mecanismo cuyo único fin era el abuso del poder. El autor que se encuentra de trasfondo es Thomas Hobbes, tamizado por la interpretación que Schmitt lleva a cabo en su libro sobre el Leviatán publicado en $1938 .{ }^{41}$ Con todo, las diferencias de fondo son notables. Schmitt lee a Hobbes desde el prisma de la teología política. En este sentido, para el jurista alemán, el sentido de la teoría política de Hobbes reside, concediendo razón en este punto a Leo Strauss, en la restitución de la unidad entre política y religión, una unidad a la que la Iglesia de Roma, amén de las iglesias presbiterianas, habría coadyuvado a aniquilar dejando de considerar la religión como parte de la política. ${ }^{42}$ Con ello Schmitt se está posicionando de manera implícita ante los argumentos que desarrolló Erik Peterson en El monoteísmo como problema político, publicado en 1935. Peterson percibía en las posiciones de la teología política schmittiana ${ }^{43}$ un intento de instrumentalizar el Evangelio para justificar una situación política concreta, ${ }^{44} \mathrm{y}$ orientaba parte de su esfuerzo a demostrar la imposibilidad teológica de toda teología política, cuyo origen se remontaría al concepto de monarquía del judaísmo alejandrino, ${ }^{45}$ mientras

40. C. Schmitt, “Zu Friedrich Meineckes..., op. cit., 233. Esta idea sobre la separación de lo público y lo privado como precondición del liberalismo burgués del siglo XIX, y que se manifiesta en la disyuntiva Estado/sociedad civil, se encontraba igualmente en Brunner. Cf. J. van Horn Melton, "From Folk History to Structural History: Otto Brunner (1898-1982) and the Radical-Conservative Roots of German Social History”, en J. van Horn Melton y H. Lehmann (Dir.), Paths of Continuity, Cambridge, 1994, 263-292, aquí: 272.

41. C. Schmitt, Der Leviathan in der Staatslehre des Thomas Hobbes, Stuttgart, 2015.

42. C. Schmitt, Der Leviathan... op. cit., 21.

43. El primero de los dos ensayos de Schmitt sobre teología política fue publicado en 1922.

44. E. Peterson, El monoteísmo como problema político, Madrid, 1999, 95.

45. E. Peterson, El monoteísmo... op. cit., 62. No obstante, Schmitt, especialmente en su segundo ensayo sobre teología política, publicado en 1970, pareció haber comprendido de manera unilateral el tratado de Peterson sobre el monoteísmo político, reduciéndolo al intento por liquidar su propuesta de teología política cuando, en realidad, el punto de referencia de ese tratado no era otro que la teología imperial (Reichstheologie). Cf. a este respecto B. Nichtweiss, Erik Peterson. Neue Sicht auf Leben und Werk, Friburgo, 1994, 810. 
que Schmitt veía que «la liquidación de la teología política por parte de la teología -el caso Peterson- no es sino la apertura de otra política, la que deslegitima al Estado como instancia central, soberana, total y cuestiona la sociedad homogénea, el pueblo que lo sostiene.» ${ }^{46}$ En este sentido, Schmitt percibía que tal intento de liquidación no era sino la prueba de que la teología había sucumbido al liberalismo. Quizá por ello su reedición de la teología política no sería más que el propósito, cual plan de emergencia, de salvar «una estructura de poder sin vida, sin espíritu y sin futuro». ${ }^{47}$

El cometido de Koselleck no era, empero, escudriñar la urdimbre de la teología política. Para él, la importancia de Hobbes radicaba en haber puesto sobre la mesa una solución al conflicto de las guerras de religión. Dado que los Estados confesionales, tomando a Francia como ejemplo, son incapaces de mantener el orden civil, se ha de «privatizar» la convicción moral y dejarle a la política únicamente el tener que velar por la paz social. Y es aquí donde se encuentra la primera escisión moderna, la escisión entre moral y política, que conducirá a la autonomía de la subjetividad. En su tesis doctoral, Koselleck denomina a este fenómeno «el giro hacia la modernidad» (die Wende zur Neuzeit). ${ }^{48}$ El giro hacia la modernidad seguirá siendo decisivo en el pensamiento de Koselleck aún años más tarde. Téngase en cuenta que el objetivo último de las cuatro «hipótesis» ${ }^{49}$ que vertebran los Geschichtliche Grundbegriffe es interrogarse sobre este giro hacia la modernidad. Es más, los

46. J. L. Villacañas, “La leyenda de la liquidación de la teología política”, en Carl Schmitt, Teología política, Madrid, 2009, 135-180, aquí: 169.

47. J. L. Villacañas, Teología política imperial y comunidad de salvación cristiana, Madrid, 2016, 599.

48. R. Koselleck, Kritik und Krise. Eine Untersuchung... op. cit., 20 y 46. Resulta llamativo que tal expresión no aparezca en la publicación de la tesis. En dicha publicación encontramos una formulación similar «entscheidende Wendung», pero en un estado de cosas algo distinto al de la tesis doctoral, pues los pasajes de esta última donde aparece «Wende zur Neuzeit» no se encuentran en la publicación. Cf. R. Koselleck, Kritik und Krise. Eine Studie... op. cit., 68. En la nueva formulación para la publicación de su tesis doctoral Koselleck podría estar jugando con el sentido etimológico del concepto crisis ( $\kappa \rho \iota \iota \varsigma)$ en cuanto separación (Scheidung) o decisión (Entscheidung), tal como él mismo desarrollará años más tarde en la voz correspondiente a este concepto en el gran diccionario. Cf. el artículo "Krise” en O. Brunner, W. Conze y R. Koselleck (Dir.), Geschichtliche Grundbegriffe. Vol. 3 Stuttgart, Klett-Cotta, 1982, 617-650, aquí: 617-618. Por último, es necesario señalar también una formulación semánticamente similar: «el proceso de transformación hacia la modernidad» (Umwandlungsprozeß zur Moderne), expresión ésta que aparece en R. Koselleck, "Einleitung”, en O. Brunner, W. Conze y R. Koselleck (Dir.), Geschichtliche Grundbegriffe. Vol. 1, op. cit, XV-XVIII, aquí: XIX.

49. En el séptimo volumen del gran diccionario Koselleck llama hipótesis a lo que en el primer volumen era considerado como criterios. Sobre el término «criterio» cf. R. Koselleck, "Einleitung", op. cit; sobre el término «hipótesis» véase Reinhart Koselleck, "Volk, Nation, Nationalismus, Masse”, en O. Brunner, W. Conze y R. Koselleck (Dir.), Geschichtliche Grundbegriffe. Vol. 7, op. cit., 141-431, aquí: 147. Los cuatro criterios considerados posteriormente como hipótesis son democratización, temporalización, ideologización y politización. 
conceptos del Lexikon dan cuenta de este giro en la medida que son capaces de registrar los cambios estructurales, haciendo avanzar la historia en este proceso de registro. ${ }^{50}$

Al principio del tercer capítulo de la tesis expone también de forma sintética el planteamiento que desarrolla a lo largo del libro. En esta ocasión, el planteamiento es expuesto de tal modo que la influencia de Karl Löwith queda reflejada de manera nítida: el abismo existente entre el sentimiento de superioridad moral de la burguesía y su carencia de poder político real en el Estado absolutista fue superado mediante la construcción teórica de una filosofía de la historia que dejaba entrever el dominio al que se aspiraba como un resultado inevitable de la historia futura. ${ }^{51}$ El elemento nuclear de su planteamiento es, por tanto, el ataque político de la Ilustración al Estado absolutista con medios morales que parten de pretensiones que van más allá del ámbito político. La burguesía oculta detrás de la máscara de un ideal de humanidad su ansia de llegar al poder por interés de clase. Aquí es donde reside, según Koselleck, la hipocresía de la Ilustración: ésta dirige sus críticas al Estado absolutista en nombre de la humanidad en general, cuando, en realidad, lo que quiere es imponer sus intereses particulares. Se trata, en el fondo, de una clase social con pretensiones e intereses que no puede realizar con la situación de inferioridad política en la que se encontraba a mediados del siglo XVIII. Y es en este momento en el que nace la filosofía de la historia como una suerte de "grito de la criatura oprimida». ${ }^{52}$ Hablar de filosofía burguesa de la historia resultaría, por tanto, tautológico, ya que la filosofía de la historia sería fruto de la inferioridad política de la burguesía bajo el Estado absolutista.

\section{La recepción de Crítica y crisis}

En cuanto a la recepción de Crítica y crisis, no deja de resultar llamativa la percepción de Koselleck sobre la circunstancia de que la versión impresa de su tesis, según sus propias palabras, «se nutrió en buena medida de la influencia de Hannah Arendt sobre los orígenes y los elementos de la dominación totalitaria, pero de esto, quizá porque fui catalogado de schmittiano, nadie se dio o quiso darse cuenta». ${ }^{53}$ Koselleck quiere, en cierto modo, defenderse o, al menos, poner límites a la recepción de su obra como una prolongación de las tesis schmittianas. A este respecto recuerda que: «A quien mostraba su agradecimiento a Carl

50. R. Koselleck, "Volk, Nation, Nationalismus, Masse”, op. cit., 147.

51. R. Koselleck, Kritik und Krise. Eine Studie... op. cit., 108. Cf. H. Joas, "Die Kontingenz der Säkularisierung. Überlegungen zum Problem der Säkularisierung im Werk Reinhart Koselleck”, en H. Joas y P. Vogt (Dir.), Begriffene... op. cit., 319-338, aquí: 328.

52. Extrapolamos al origen de la filosofía de la historia la expresión que Marx formula en referencia a la esencia de la religión en su Crítica a la filosofía del derecho de Hegel: K. Marx, "Zur Kritik der Hegelschen Rechtsphilosophie”, en K. Marx y F. Engels, Werke. Vol. 1, Berlín, 1976, 378-391, aquí: 378. Sobre la filosofía de la historia (su pasado, presente y lo que pueda ser su futuro) véase A. Gómez Ramos, Reivindicación del centauro. Actualidad de la filosofía de la historia, Madrid, 2003.

53. R. Koselleck, “Dankrede..., op. cit., 53. 
Schmitt, se le consideraba su vocero, a quien citaba teorías de la conspiración del siglo XVIII, se le consideraba un teórico de la conspiración. Del mismo modo que a quien criticaba un dualismo inspirado política o moralmente se le consideraba un dualista». ${ }^{54}$ En alguna ocasión llegó a señalar que las lecturas reduccionistas de su obra le supusieron la pérdida de su primera cátedra en la Universidad de Constanza: «mi nombre -como también el de Hans Blumenberg- fue tachado de la lista de los aspirantes entre otras razones con el argumento de que era "schmittiano", después de tener ya en el bolsillo la aceptación de mi candidatura por parte de la Facultad.» ${ }^{55}$

Muy probablemente esté en lo cierto Koselleck al llamar la atención sobre lo inapropiado e injusto de reconocer en su tesis doctoral únicamente la huella de Schmitt, pasando así por alto la presencia de otros autores a lo largo de su investigación. Sin embargo, no parece posible negar que este trabajo, en el que traza las líneas del nacimiento del pensamiento político burgués y de la filosofía de la historia en la época ilustrada, se mueva dentro de las coordenadas que Schmitt buscaba establecer entre filosofía de la historia y teología de la historia. ${ }^{56}$ Por aquel entonces, las tesis de Schmitt al respecto carecían de un fundamento firme. ${ }^{57}$ Asimismo, su producción intelectual se encontraba estancada desde 1949, a parte de su librito Hamlet o Hécuba y la Teoría del partisano no publicó ningún texto destacado a partir de ese año. ${ }^{58}$ Schmitt confía esta tarea a su nueva generación de discípulos, entre los que

54. R. Koselleck, “Dankrede...”, op. cit., 55. En este punto Koselleck esté pensando quizás en la dura crítica de Habermas en "Verrufener Fortschritt - verkanntes Jahrhundert. Zur Kritik an der Geschichtsphilosophie”, en Merkur, 1960, 468-477. En los primeros párrafos de la recensión de Habermas a Crítica y crisis la argumentación para situarlo en las coordenadas teóricas de Schmitt es relativamente velada (p. 472), pero las últimas líneas son una acusación directa (tanto a Koselleck como a Hanno Kestin): «Immerhin sind wir dankbar, von so gescheiten Autoren zu erfahren, wie Carl Schmitt, ein so denkender Spezialist, die Lage heute beurteilt.» (p. 477).

55. "Formen der Bürgerlichkeit. Reinhart Koselleck im Gespräch mit Manfred Hettling und Bern Ulrich", en Mittelweg 36, 2, 2003, 75. Citado por F. Oncina, "Necrológica del Outsider Reinhart Koselleck: el 'historiador pensante’ y las polémicas de los historiadores”, en Isegoría, 37, 2007, 35-61, aquí: 41, nota 13.

56. De hecho el propio Schmitt publicó una recensión sobre Crítica y crisis: C. Schmitt, "Rezension von Koselleck, Kritik und Krise”, en Das Historisch-politische Buch, 1959, 301-302. Los dos esbozos de esta recensión se encuentran en el Nachlass Carl Schmitt, junto a una carta que Schmitt le envió a Koselleck el 21/06/1959. Si bien hay variaciones entre ambos esbozos, la tesis fundamental no varía, a saber: «Crítica y crisis no es un libro de historia de las ideas al estilo de la Idea de razón de Estado de Meinecke», C. Schmitt a R. Koselleck, en RWN 260-386. Koselleck finalmente se decantó por el segundo esbozo. Cf. R. Mehring, "Begriffsgeschichte mit Carl Schmitt", en H. Joas y P. Vogt (Dir.), Begriffene Geschichte, op. cit., 138-168, aquí: 140 , nota 15 .

57. R. Mehring, Carl Schmitt... op. cit., 492.

58. R. Mehring, Carl Schmitt... op. cit., 505. Se hace necesario en este punto llamar la atención sobre el hecho de que Schmitt se encargó de redactar el prólogo a la traducción que su hija Anima hizo del libro de Lilian Winstanley titulado Hamlet and the Scottish Succession, publicado en 1921. La traducción de Anima Schmitt, con el prólogo de su padre, se publicó en 1952 con el título Hamlet, Sohn der Maria Stuart; publicación datada, pues, cuatro años antes de que Schmitt publicase su libro sobre Hamlet. De Koselleck 
destacan especialmente Nicolaus Sombart, Hanno Kesting y el propio Koselleck. «Lo que por aquel entonces se estaba considerando era la disyunción entre filosofía de la historia y teología de la historia para llevar a cabo de manera fructífera una crítica ideológica del utopismo del progreso y asegurar así un nuevo terreno a la crítica universalista.» ${ }^{59}$

El propio Koselleck dedica su tesis doctoral a Carl Schmitt, «quien, como es notorio, además de subrayar la importancia de la literatura del arcano, imputa a la Ilustración -al carácter pernicioso de la publicidad- y a la Revolución la presunta responsabilidad de las patologías modernas. Este lastre schmittiano motiva el repudio de las tesis del libro por parte de Habermas en su reseña Crítica a la filosofía de la historia.» ${ }^{60}$ Con este tipo de afirmaciones Koselleck no se lo puso difícil a Habermas. El máximo representante de la segunda generación de la Escuela de Fráncfort bien podía ya redactar su particular pliego de cargos. Y quizá no le falte razón cuando echa en falta un apartado dedicado a la figura de Koselleck en el libro de Dirk van Laak sobre la presencia de Carl Schmitt en la historia de la cultura política de los comienzos de la República Federal de Alemania, ${ }^{61}$ pudiendo así situarlo bajo la égida del conservadurismo revolucionario abanderado por el jurista de Plettenberg. «Habermas, por otra parte, parece no ser consciente de que su Historia y crítica de la opinión pública. La transformación estructural de la vida pública (1962) tiene una importante deuda con Koselleck.» ${ }^{62}$ De hecho, los análisis de Habermas sobre la evolución de lo público a lo largo de la historia -en una constante dialéctica con el espacio de lo privado, lo que le permite caracterizar el concepto de opinión pública en el proceso de ascenso político de la burguesía- difícilmente podrán entenderse sin la referencia a Crítica y crisis. Téngase en cuenta la cercanía temporal de ambos escritos: Koselleck defiende su tesis en 1954, la publica en 1959 y la primera edición del libro de Habermas es de 1962. En el plano de los elementos concretos pueden verse, por ejemplo, las referencias a la masonería ${ }^{63}$ a través de la figura de Lessing que se encuentran en el apartado V del capítulo II de Historia y crítica de la opinión pública para tratar la problemática de las instituciones de la publicidad como un ideal democrático que emerge en el

se conserva un esbozo de recensión a esta traducción de Anima Schmitt: R. Koselleck, "Hamlet, Sohn der Maria Stuart”, en H. Joas y P. Vogt (Dir.), Begriffene... op. cit., 169-170.

59. R. Mehring, Carl Schmitt... op. cit., 492. Respecto al concepto de utopía y utopismo en Koselleck, en una reciente publicación afirma Faustino Oncina que «la utopía depende de la posibilidad de ser de otra manera, pero el utopismo es inexorablemente totalitario en la medida en que entraña la abolición de la contingencia, de la inconmensurabilidad en los humanos entre deseo y realización, intención y realidad. Aun admitiendo la necesidad de matizaciones, despacha al "utopista" como "alguien que quiere de forma insensata algo irreal y no factible" y condena a los hipotéticos "utopianos" a sufrir los engendros monstruosos de sus sueños.» F. Oncina Coves, "Utopías y ucronías en la teoría de los tiempos históricos de la historia conceptual”, en F. Oncina y J. de Dios Bares (Dir.), Utopías y ucronías. Una aproximación histórico-conceptual, Barcelona, 2020, 13-38, aquí: 18.

60. R. Koselleck/H.-G. Gadamer, Historia y hermenéutica, Barcelona, 1997, 78, nota 11.

61. D. van Laak, Gespräche... op. cit., 1993.

62. F. Oncina, "Historia conceptual y hermenéutica”, en Azafea, 5, 2003, 161-190, aquí: 184, nota 47.

63. J. Habermas, Historia y crítica de la opinión pública. La transformación estructural de la vida pública, Barcelona, 1982, 73. 
encuentro con el privilegio soberano del secreto y del que la masonería sería la realización práctica de ese ideal. ${ }^{64}$ Tema, éste, central, sin duda, del primer libro de Koselleck. Sin olvidar tampoco la reconstrucción crítica que lleva a cabo Habermas de los argumentos que usa Hobbes para captar la función de la opinión pública recurriendo al concepto de soberanía. ${ }^{65}$ Téngase en cuenta en este punto el papel que juega para Koselleck el concepto de soberanía vinculada estrechamente a la noción de sujeto hobbesiano entendiendo éste como un sujeto escindido en dos esferas: la esfera política externa referida a las acciones del sujeto que actúa bajo la ley (el «ciudadano») y la esfera interna de la conciencia (el «hombre»). Es en esta última esfera donde se encuentra uno de los grandes arcanos de la masonería, a saber, que la libertad en secreto es el secreto de la libertad. ${ }^{66}$ Resulta asimismo llamativo que esta temática de la tesis doctoral de Koselleck llega incluso a dar título a uno de los apartados cruciales de Historia y crítica de la opinión pública: Se trata del primer apartado del capítulo V titulado «La tendencia al ensamblamiento de esfera pública y ámbito privado».

La separación entre la esfera interna y la esfera externa del sujeto podría ser muy probablemente el caballo de batalla de toda interpretación política de la obra Hobbes. El uso conceptual que de esa separación hace Koselleck encuentra una referencia directa en el libro de Schmitt sobre Hobbes, donde habla específicamente de esa «separación entre lo interno y lo externo»; ${ }^{67}$ e incluso llega a considerar que «la separación entre el dentro y el afuera, lo privado y lo público, no dominaba no sólo el pensamiento jurídico, sino que correspondía a la convicción general de todos los eruditos.» ${ }^{68}$ La problemática de la interioridad -o, más específicamente, la «soberanía de la interioridad»- es, en todo caso, una cuestión que se remonta, cuanto menos, a la teología paulina en tanto en cuanto se trata de un punto cardinal de la «nueva religión de salvación». ${ }^{9}$

\section{Toma de contacto con Carl Schmitt}

El reconocimiento público de la deuda con Carl Schmitt muestra sobre todo el arrojo ante el fariseísmo de una intelectualidad alemana que se nutría en buena medida de los textos de Carl Schmitt sin reconocer abiertamente su herencia. «Manifestar en el Prólogo mi agradecimiento a Schmitt - reconoce Koselleck- fue ya un acto de coraje. Pues apenas nadie se había atrevido por aquel entonces a hacer algo así, aunque todos sus escritos fueron leídos

64. Cf. J. Dean, “Publicity’s Secret”, en Political Theory, 5, 2001, 624-650, aquí: 626.

65. J. Habermas, Historia... op. cit., 118.

66. R. Koselleck, Kritik und Krise. Eine Studie... op. cit., 60.

67. C. Schmitt, Der Leviathan... op. cit., 87.

68. C. Schmitt, Der Leviathan... op. cit., 91

69. J. L. Villacañas, Teología... op. cit., 200. Villacañas abunda en este aspecto argumentando que el griego estaba «mucho más volcado a la exterioridad de un cosmos sagrado en sí mismo. Pablo [...] reinicia la racionalización ética del griego, pero al hacerlo culmina la desacralización del cosmos.» (op.cit.., 201). 
y discutidos. $\gg^{70} \mathrm{Y}$ es que, desde la derrota del nacionalsocialismo, Carl Schmitt estaba en el punto de mira por su simpatía e implicación ideológica con el régimen, especialmente en los textos publicados alrededor de los años 30. ${ }^{71}$ Tras finalizar la II Guerra Mundial, pierde la cátedra que ostentaba en Berlín. El 30 de abril de 1945, día en el que Hitler se casa y se suicida, Schmitt es temporalmente arrestado e interrogado por militares soviéticos en su casa de la capital alemana. ${ }^{72}$ El 26 de septiembre de ese mismo año es arrestado por tropas estadounidenses y llevado al centro de interrogatorios de Berlín-Wannsee. Pasado un mes, el 31 de octubre de 1945, es trasladado al campo de internamiento Berlín/Lichterfelde-Süd. A partir de esta fecha y hasta el 10 de octubre de 1946, Schmitt pasará por varios campos de internamiento. ${ }^{73}$ En mayo de 1947, por fin liberado, se retira a Plettenberg, su ciudad natal situada en la región de Renania del Norte Westfalia. Desde esa fecha y hasta finales de 1950 será un periodo de búsqueda y restablecimiento de contactos, amén de la inseguridad económica, en la vida del jurista alemán. Hasta la fundación de la República Federal de Alemania (23 de mayo de 1949) Schmitt tenía prohoibido publicar. ${ }^{74}$ «Su pasado nacionalsocialista no se había olvidado. Ya no podía retomar su carrera académica; como autor tenía ciertas dificultades y como conferenciante ya no levantaba tanta expectación.» ${ }^{75}$

En 1950, año en el que fallece su mujer en la Krehl-Klinik de Heidelberg, Schmitt comienza a fortalecer los contactos con jóvenes talentos. Fue en ese mismo año cuando entabló por primera vez contacto personal con Koselleck. ${ }^{76}$ Por aquella época los vínculos intelectuales se establecían más allá de las facultades particulares, y Heidelberg fue buen ejemplo de ello. ${ }^{77}$ El círculo de amigos surgido de los estudiantes que frecuentaban el Privatissimum de Alfred Weber en Heidelberg que ya estaban en contacto con Carl Schmitt, y cuya relación procuraban cuidar y fortalecer, fue el punto de contacto entre Koselleck y el jurista alemán. Especialmente importantes, dada su labor mediadora, fueron las figuras de los sociólogos Hanno Kesting y Nicolaus Sombart, ${ }^{78}$ que posteriormente serían también los jóvenes más

70. “Formen der Bürgerlichkeit... op. cit., 75. Citado por Faustino Oncina, “Necrológica..., op. cit., 41, nota 13 .

71. Cf. J. W. Bendersky, "Carl Schmitt and Nuremberg”, en Telos: Critical Theory of the Contemporary, 1987, 91-96.

72. R. Mehring, Carl Schmitt... op. cit., 438.

73. R. Mehring, Carl Schmitt... op. cit., 443.

74. R. Mehring, Carl Schmitt... op. cit., 470.

75. R. Mehring, Carl Schmitt... op. cit., 504.

76. Cf. R. Koselleck, “Dankrede...”, op. cit., 34-35.

77. S. P. Remy, The Heidelberg Myth: The Nazification and Denazification of a German University, Cambridge, 2003. Sobre la presencia e influencia de Schmitt en los círculos universitarios de Heidelberg véanse especialmente las páginas 222-223; así como D. van Laak, Gespräche... op. cit., 186-192. Sobre la relación de Schmitt con el Collegium Philosophicum de Münster véase H. Ritter, Verehrte Denker: Porträts nach Begegnungen, Springe, 2012, 7-9; y, de nuevo, D. van Laak, Gespräche... op. cit., 192-200.

78. D. van Laak, Gespräche... op. cit., 188 y 272; así como R. Mehring, “Begriffsgeschichte...” op. cit, 150. 
representativos dentro del círculo que se formó en torno a Schmitt y que frecuentarían el Privatissimum de Plettenberg. ${ }^{79}$

La figura de Schmitt fue, sin duda, decisiva, y no solo en los primeros escritos de Koselleck, sino también en el monumental diccionario Geschichtliche Grundbegriffe. De hecho el elenco de categorías que introducen como reacción contra la filosofía de la conciencia y contra la metafórica especular se ve incluso desplazado por la fascinación que Carl Schmitt -con un sino común al heideggeriano- ejerce entre quienes profesan la Begriffsgeschichte en Alemania. Allá por los años 50 el círculo de Joachim Ritter en Münster, con Hermann Lübbe como miembro aventajado, y el «club de fans» schmittianos surgido de los alumnos disidentes del sociólogo Alfred Weber en Heidelberg, entre ellos Reinhart Koselleck, que años más tarde creó su propio entorno en Bielefeld (cuya universidad fue fundada a instancias de la política académica de Lübbe, secretario de Estado de Renania Westfalia del Norte, donde él mismo recaló efímeramente [1969-1971] tras su abandono del Ministerio), representan pruebas rotundas de esa hiedra conservadora de la que tanto ha recelado y continúa recelando Habermas. ${ }^{80}$

Sin duda alguna, Koselleck «ha prodigado sin rebozo sus alusiones al jurista luciferino como precursor de su Begriffsgeschichte.» ${ }^{81}$ En una conversación con Christof Dipper, Koselleck no se anda con rodeos a la hora de reconocer la impronta de la metodología schmittiana en su obra:

Carl Schmitt fue uno de los grandes estímulos, siempre llamaba la atención sobre las consecuencias de las acciones políticas en los conceptos jurídicos. Él siempre me exigía que comparase enciclopedias y me preguntaba constantemente: ¿qué significaba un término en una época concreta, ¿dónde, qué y para quién? Y esta rigorosa pregunta la había expuesto él mismo de manera brillante en su escrito sobre la dictadura. ${ }^{82}$

Puede apreciarse, pues, el papel decisivo que desempeñó Schmitt en la configuración del pensamiento de Koselleck y en su práctica de la historia conceptual. Se trata de una deuda que perduraría a lo largo de los años, lo cual él mismo, como se acaba de mostrar, nunca dejó de reconocer. En siguiente apartado seguirá abundando en la relación entre Koselleck y Schmitt desde la correspondencia entre ambos.

79. N. Sombart, Rendezvous..., op. cit., 203.

80. J. L. Villacañas y F. Oncina, "Introducción”, en R. Koselleck/H.-G. Gadamer, Historia y hermenéutica, op. cit., 9-54, aquí: 23

81. F. Oncina, "Historia..., op. cit., nota 47.

82. R. Koselleck/Ch. Dipper, "Begriffsgeschichte, Sozialgeschichte, begriffene Geschichte", en Neue politische Literatur, 43, 1998, 187-205, aquí: 187. Cf. R. Koselleck, "Begriffsgeschichtliche Probleme der Verfassungsgeschichtsschreibung”, en Begriffsgeschichten, Fráncfort del Meno, 2006, 365-401. 


\section{La presencia de Schmitt y la intrahistoria de la tesis doctoral}

Como se señaló al inicio de este ensayo, el intercambio de cartas entre ambos autores que se encuentra en el Nachlass Carl Schmitt comienza el 21 de enero de 1953 y termina el 20 noviembre 1983. Si bien la asiduidad de la correspondencia va menguando considerablemente a partir del momento en que Koselleck obtiene la plaza de catedrático - primero en la facultad de ciencias políticas de la Universidad de Bochum (1966-1967), después en la facultad de historia en la Universidad de Heidelberg (1968-1973) y finalmente en Bielefeld (1974-1988) donde ocupará la cátedra de Teoría de la historia ${ }^{83}$ ambos autores mantendrán una relación más que cordial hasta el fallecimiento del jurista alemán. Al abrir los archivos de esta correspondencia, lo primero que salta a la vista no es precisamente una carta, sino una postal que Koselleck envía a Schmitt desde Escocia con una imagen del Glenfinnan Monument, monumento que conmemora la causa jacobita en la rebelión de 1745 que intentaba restaurar por tercera vez a la familia Estuardo en el trono de Gran Bretaña.

En la primera carta, con fecha del 21 de enero de 1953, Koselleck agradece a Schmitt su hospitalidad por haberle recibido y acogido en su casa de Plettenberg; asimismo, muestra su agradecimiento por la deferencia al haber leído detalladamente el borrador de su tesis doctoral. ${ }^{84}$ Koselleck, además de fascinado, se siente intelectualmente deudor de Schmitt. Un fragmento de esa primera carta representa de manera ejemplar el acercamiento intelectual al jurista de Plettenberg. Aunque no sólo eso. En ella aparece ya in nuce diferentes aspectos propios de la historia conceptual que Koselleck irá desarrollando con el paso de los años.

El componente emocional y de admiración hacia Schmitt que se manifiesta en los primeros años de correspondencia marca el tono de la escritura. Koselleck deja entrever asimismo la incertidumbre respecto a su futuro. Entre ambos se crea una atmósfera de cordialidad muy especial. En noviembre de 1953, el joven historiador se encuentra en Bristol, Schmitt ha leído su tesis, no por encima, sino durchgelesen, y se reserva las sugerencias para un encuentro en Plettenberg que Koselleck le propone para poco antes de la Navidad de ese mismo año. La simpatía de Schmitt por Koselleck y la afinidad con el tema de su tesis doctoral resultan evidentes. En una carta fechada el 11/11/53 Schmitt le escribe a Koselleck:

Su tesis doctoral ha superado la travesía de Bristol a Plettenberg y ha llegado aquí con éxito. La he recibido con gran alegría y acabo de mantener una excelente conversación con ella [...] Ya la he revisado y me he preparado una serie de notas para nuestra conversación de enero que con tantas ganas espero ${ }^{85}[\ldots]$ En este momento la pregunta que más me ocupa es la de saber cómo van a afrontar los examinadores de Heidelberg este extraordinario trabajo. ¿Le dirán a

83. N. Olsen, History in the plural. An Introduction to the Work of Reinhart Koselleck, New York/Oxford, 2012, 6 .

84. R. Koselleck a C. Schmitt, 21/01/1953, en RW 265-8131. Ahora en R. Koselleck/C. Schmitt, Der Briefwechsel... op. cit., 9-17, aquí: 9

85. La cita estaba prevista para enero de 1954. Al adelantarse la defensa de la tesis a esa fecha, Koselleck le propone a Schmitt el encuentro para la Navidad de 1953. 
usted, siguiendo a Lessing, sabes que ya eres medio masón? ${ }^{36}$ ¿Van a consideran su trabajo como la violación de un tabú cuando se toca el dualismo entre política y moral y no verán en ello sino una amenaza para su propia existencia intelectual? ${ }^{87}$

Puede apreciarse aquí claramente que, como se señaló anteriormente, el concepto Dualismus von Moral und Politik del que se servirá Koselleck para sustituir el de moralischer Dualismus procede igualmente del aparato conceptual de Carl Schmitt. ${ }^{88}$ Desde Bristol, Koselleck responde parcialmente a estas preguntas unas semanas más tarde, el 29 de noviembre de 1953, no sin cierta molestia por esa referencia a Lessing:

La advertencia de Lessing no me la hará nadie. A lo que sí podrían referirse es a la supuesta neutralidad de una metodología científica para poder reprocharme una falta de cientificidad en nombre de un planteamiento metodológico diferente que corresponda a esa neutralidad. Y, sin duda, tácticamente me he ajustado demasiado poco a ello. El profesor Kühn es tan tolerante que puede admitir mi planteamiento del problema, pero me temo que con este trabajo he agotado hasta tal punto su tolerancia que no daría un paso más allá aunque fuese necesario [... Habrá que escuchar también la exposición del profesor Löwith, cuyo escepticismo en torno a la filosofía de la historia - ja no ser que fuese cosa de la emigración!- no entra en absoluto en contradicción con mi trabajo. ${ }^{89}$

En enero de 1954 Koselleck ha de defender su tesis doctoral. La tesis está ya entregada en Heidelberg para la lectura de los evaluadores (Johannes Kühn, su director, y Karl Löwith), pero el aún doctorando reside en Bristol, cuidad en la que trabaja como lector, tiempos en los que la carrera académica aún quedaba lejos. Tal como se aprecia en el fragmento de la carta de Schmitt, el encuentro en Plettenberg estaba acordado en un primer momento para enero del 54, pero Koselleck envía una carta al jurista alemán pidiéndole adelantarlo:

Dado que es muy poco probable que la defensa de la tesis tenga lugar antes de Navidad y que, por otro lado, el examen quizá sea en la segunda semana de enero, querría preguntarle, muy estimado profesor, si, en lugar de en enero, podría visitarle poco antes de Navidad. Entre el 17 y el 20 de diciembre pasaré por Westfalia para ir a casa y le estaría muy agradecido si me escribiese

86. Schmitt se refiere al texto Ernst y Falk. Diálogo para masones: «¿Sabes, amigo, que ya eres medio masón?» (Weißt du, Freund, daß du schon ein halber Freimäurer bist?), en Gotthold Ephraim Lessing, Werke. Vol. 8, Múnich, 1970, 458.

87. C. Schmitt a R. Koselleck, 11/11/1953, en RWN 260-386. Ahora en R. Koselleck/C. Schmitt, Der Briefwechsel... op. cit., 31. La cursiva en la cita es nuestra.

88. Cf. notas 34 y 35.

89. R. Koselleck a C. Schmitt, 29/11/1953, en RW 265-8134. Ahora en R. Koselleck/C. Schmitt, Der Briefwechsel... op. cit., 34-35, aquí: 34 . 
confirmándome si le puedo visitar uno de esos días. Si el examen se programara para enero, tal vez iría muy justo de tiempo, ya que el semestre aquí comienza el 15 de enero. ${ }^{90}$

Koselleck defiende finalmente la tesis en Heidelberg el mes de enero del 54, pero el futuro aún es incierto. El recién doctorado muestra a Schmitt sus dudas e inquietudes sobre el porvenir de un modo bastante explícito. ¿Sigue adelante en Bristol o vuelve a Alemania? Ideas para investigar, desde luego, no le faltan. En una carta fechada el 14/11/1954 escribe:

La pregunta que debo elaborar próximamente está relacionada con mi puesto de aquí en Bristol. Mi profesor de aquí me ofreció un segundo año de lectorado. Si acepto mantener el puesto en esta universidad, un puesto que a la larga no es precisamente apasionante, tendría la posibilidad, relativamente bien pagada, de tratar un tema inglés en amplios periodos de tiempo libre y en las vacaciones pagadas. Es posible que el tema lo pueda continuar en Estados Unidos, donde tal vez podría obtener una beca. En los dos años siguientes podría intentar investigar, siguiendo la línea trazada en el trabajo que he realizado hasta ahora, la filosofía del progreso (y la filosofía cíclica) del siglo dieciocho con sus implicaciones política e históricas [...] Naturalmente, habría aún una gran cantidad de temas que, según creo, no han sido expuestos de manera adecuada. Por ejemplo, la creciente democratización de Inglaterra y el correspondiente papel del Imperio, o Disraeli y Marx. ${ }^{91}$

Meses más tarde, el joven Koselleck dejará a un lado estas inquietudes sobre su futuro en el mundo anglosajón al recibir una propuesta para trabajar en el Instituto para la Investigación Social (Sozialforschungstelle) fundado por la Universidad de Münster, y cuya sede se encuentra a unos setenta kilómetros de distancia, en la ciudad de Dortmund. En una carta del 6 de enero de 1955 escribe: «Sobre mi futuro aún no sé nada. El señor Popitz se había esforzado por encontrarme un posible trabajo en el Instituto de Dortmund y precisamente recibí una invitación del profesor Ipsen para ir a hablar con él a final de mes.» ${ }^{92}$ Koselleck ve con buenos ojos la propuesta de trabajar en el Instituto de Dortmund, ya que esto le permitiría investigar, desde una perspectiva más sociológica, sobre temas actuales. Sin embargo, hay algo que no le convence del todo. Se trata precisamente de profundizar en esa perspectiva sociológica. El joven investigador se plantea cuánto le alejaría ese puesto de una futura carrera como historiador.

Koselleck acaba abandonando Bristol de manera definitiva y con ello los proyectos que le supondrían afincarse e intentar hacer carrera en el mundo anglosajón. Acepta el puesto en Dortmund, pero poco después lo rechaza por una plaza de asistente con Johannes Kühn en

90. R. Koselleck a C. Schmitt, 29/11/1953, en RW 265-8134. Ahora en R. Koselleck/C. Schmitt, Der Briefwechsel... op. cit., 34-35.

91. R. Koselleck a C. Schmitt, 14/11/1954, en RW 265-8135. Ahora en R. Koselleck/C. Schmitt, Der Briefwechsel... op. cit., 43.

92. R. Koselleck a C. Schmitt, 06/07/1955, en RW 265-8141. Ahora en R. Koselleck/C. Schmitt, Der Briefwechsel... op. cit., 90-101, aquí: 95. 
Heidelberg. En una carta fechada el 28/08/1955, esta vez enviada no desde Bristol sino desde la ciudad de Hannover, de cuya Pädagogische Hochschule era docente su padre, le habla a Schmitt del nuevo cambio:

De repente, tras mi vuelta de Dortmund, me encontré en casa en una difícil situación. Al llegar tenía una carta del profesor Kühn en la que me animaba a incorporarme inmediatamente a su puesto de asistente. En cierto modo, estaba obligado a cometer al menos una tontería. Al final, tras considerarlo detenidamente, me decidí a aceptar el puesto de Heidelberg y pasar el mal trago de rechazar el de Dortmund una vez aceptado [...] Pero mientras siga aspirando a un habilitación en historia, el acceso a Heidelberg me parece más llano, a pesar de que me expongo a la incertidumbre de lo que sucederá cuando el Profesor Kühn le deje su cátedra a un todavía desconocido sucesor. ${ }^{93}$

El sucesor de Johannes Kühn sería finalmente Werner Conze, lo cual le supuso, pasado el tiempo, que su trabajo de habilitación debiera moverse dentro de las coordenadas de una historia social que apenas dejaba espacio para la teoría de los tiempos históricos. En definitiva, si quería seguir adelante, debía reorientar, al menos en principio, la dirección de su investigación. El trabajo de habilitación era el siguiente paso. Koselleck tenía ya por aquel entonces planeado llevar a cabo un estudio comparativo de las estructuras temporales del Congreso de Viena con las del Tratado de Versalles y presentarlo como trabajo de habilitación. Werner Conze tenía, sin embargo, otros planes para él. La dimensión histórico-conceptual del Congreso de Viena no encajaba del todo con el grupo de investigación que Conze había formado en Heidelberg en 1957. El sucesor de Kühn le ofreció escribir un libro sobre historia del derecho de Prusia que se convertiría posteriormente en su trabajo de habilitación gracias al cual obtuvo la venia legendi..$^{94} \mathrm{He}$ aquí las palabras del propio Koselleck años más tarde sobre las que, a la sazón, eran las nuevas directrices de su investigación:

La pregunta por los tiempos históricos se encontraba ya implícita en mi tesis doctoral [...] Antes de que Werner Conze llegase a Heidelberg como mi segundo maestro, intenté escribir un trabajo de habilitación que debía comparar las estructuras temporales del Congreso de Viena con el Tratado de Versalles. Esto habría conducido a contrastar la antigüedad de los títulos jurídicos, su fuerza innovadora y su durabilidad en la vida política, un contraste que habría sido favorable al Congreso de Viena [...] Sin embargo, Wener Conze no tenía interés en este trabajo sobre la temporalidad en el ámbito del derecho internacional. Él me propuso el libro sobre Prusia. En él traté de manera implícita cuestiones teóricas referidas a la temporalidad sin llegar a exponerlas en la teoría. A pesar de ello, el libro sobre Prusia es un libro

93. R. Koselleck a C. Schmitt, 28/08/1955, en RW 265-8142. Ahora en R. Koselleck/C. Schmitt, Der Briefwechsel... op. cit., 101-103, aquí: 102.

94. R. Koselleck, Preußen zwischen Reform und Revolution, Stuttgart, 1989. 
teórico-temporal porque en él mido la duración de la validez del derecho y su fuerza de transformación en dimensiones temporales. ${ }^{95}$

A pesar de las diferencias iniciales entre ambos historiadores, «no es exagerado afirmar que para Conze el trabajo del que por aquel entonces fuera su asistente cumplía exactamente con lo que él había considerado que debía ser una buena historia de las estructuras. ${ }^{96}$ Con la venia legendi recibida por la investigación sobre Prusia Koselleck cumple ya los requisitos para optar a una cátedra (Professur). El primer destino en esa búsqueda será Bochum, después Heidelberg y, finalmente, la cátedra de Teoría de la historia en Bielefeld. Este nuevo cambio no supone detrimento alguno en la relación con Schmitt, si bien es cierto que aumenta la distancia temporal en la correspondencia. Koselleck, siempre respetuoso, se disculpa por estos retrasos. Trabajo, familia y, sobre todo, la burocracia universitaria que comienza a aumentar considerablemente tras ser nombrado director del Zentrum für interdisziplinäre Forschung de Bielefeld le ocupan un tiempo precioso. La relación intelectual y personal sigue igual de intensa, aunque Koselleck ya no visita a Schmitt con tanta frecuencia, ni Schmitt se presenta de manera espontánea en casa de los padres de Koselleck como lo hacía antes. Koselleck le sigue enviando textos junto con la correspondencia. Pero ya no son libros o artículos que ha leído y que podrían interesar al jurista alemán, sino que se trata de su propia producción intelectual. Artículos, conferencias, los primeros volúmenes de los Geschichtliche Grundbegriffe, la traducción española de Kritik und Krise, etc.

En todo caso, el pensamiento de Schmitt ejerció una influencia sobre el desarrollo de la historia de los conceptos de Koselleck que éste nunca disimuló. Ahora bien, esta influencia es eminentemente teórica, y no ya política. De otro modo difícilmente podrían entenderse los postulados en absoluto conservadores que arguyó Koselleck en la polémica en torno a los monumentos en conmemoración a las víctimas de la II Guerra Mundial. ${ }^{97}$ Esto no significa, sin embargo, que la relación entre el jurista y el historiador quede definitivamente libre de toda controversia.

95. R. Koselleck, “Zeit, Zeitlichkeit und Geschichte - Sperrige Reflexionen. Reinhart Koselleck im Gespräch mit Wolf-Dieter Narr und Kari Palonen”, en Zeit, Geschichte und Politik. Zum achtzigsten Geburtstag von Reinhart Koselleck, J. Kurunmäki y K. Palonen, (Dir.), Jyväskyla, 2003, 9-33, aquí: 9-10.

96. J. E. Dunkhase, Werner Conze. Ein deutscher Historiker im 20. Jahrhundert, Gotinga, 2010, 144.

97. Cf.. R. Koselleck, Modernidad, culto a la muerte y memoria nacional, Madrid, 2011. 